\title{
Aplicabilidade do desenvolvimento tecnológico nas reabilitações orais: relato de caso
}

\author{
Applicability of technological development in oral rehabilitation: case report \\ Aplicabilidad del desarrollo tecnológico en rehabilitación oral: relato de caso
}

Recebido: 19/01/2021 | Revisado: 23/01/2021 | Aceito: 25/01/2021 | Publicado: 31/01/2021

\author{
Horácio D’Aguiar Silva Belo \\ ORICD: https://orcid.org/0000-0003-1744-0363 \\ Universidade de São Paulo, Brasil \\ E-mail: horaciobeloodontologia@gmail.com \\ André Luiz Marinho Falcão Gondim \\ ORCID: https://orcid.org/0000-0002-3581-419X \\ Universidade Federal do Rio Grande do Norte, Brasil \\ E-mail: algondim@yahoo.com.br \\ Flávio Domingues das Neves \\ ORCID: https://orcid.org/0000-0002-8676-302X \\ Universidade Federal de Uberlândia, Brasil \\ E-mail: flaviodominguesneves@gmail.com \\ Andréia Aparecida Traina \\ ORCID: https://orcid.org/0000-0001-9193-1027 \\ Universidade de São Paulo, Brasil \\ E-mail: aatraina@usp.br \\ Marta Gonzalez Riesco \\ ORCID: https://orcid.org/0000-0002-9264-3075 \\ Fundação Faculdade de Odontologia - USP, Brasil \\ E-mail: mriesco2@uol.com.br \\ Katia Arduini Frata \\ ORCID: https://orcid.org/0000-0002-9109-0149 \\ Abeno-Nap-Odonto, Brasil \\ E-mail: kafrata@hotmail.com \\ Luis Ferreira de Almeida Neto \\ ORCID: https://orcid.org/0000-0003-3141-1227 \\ Universidade Federal do Rio Grande do Norte, Brasil \\ E-mail: luisneto_w@hotmail.com \\ Karolina Pires Marcelino \\ ORCID: https://orcid.org/0000-0002-2301-5155 \\ Universidade Federal do Rio Grande do Norte, Brasil \\ E-mail: karolpiresm@gmail.com \\ Ivete Aparecida de Mattias Sartori \\ ORCID: https://orcid.org/0000-0003-3928-9430 \\ Faculdade ILAPEO, Brasil \\ E-mail: ivetemsartori@gmail.com
}

\begin{abstract}
Resumo
A odontologia reabilitadora está passando por uma grande evolução em todas as áreas de conhecimento, sejam pelas pesquisas cientificas, desenvolvimento das técnicas cirúrgicas e desenvolvimento de materiais. Com o surgimento de novas tecnologias, mais recursos vem sendo adregados na clínica diária, já que materiais e técnicas auxiliam desde o diagnóstico até a confecção das próteses sobre implantes. Objetiva-se, neste relato de caso clínico, demonstrar a importância da associação de várias áreas do conhecimento na reabilitação implantossuportada, valorizando os benefícios da utilização das tomografias computadorizadas cone beam, do sistema CAD/CAM, dos scaners intraorais e das técnicas adesivas nas reabilitações. Paciente com 54 anos procurou tratamento na Fundecto-USP (Fundação Faculdade de Odontologia conveniada à FOUSP) com queixa de ausência dentária na região dos elementos 14 e 15, além de queixas referentes à estética na região anterior. Foi realizado o diagnóstico através de montagem em articulador semi-ajustável, fotografias e enceramento diagnóstico. Apesar da ausência de dois elementos, foi planejada a reabilitação com implante apenas do elemento 15 e aumento dos elementos 16 e 13 . A tomografia computadorizada cone beam, permitiu identificar ausência de altura óssea suficiente para instalação de implante na região do 15, então foi planejado a instalação do implante com levantamento da membrana do seio maxilar de forma simultânea. Através de escaneamento intraoral, tecnologia CAD/CAM e informações clínicas, foi realizado o projeto do enceramento virtual das futuras coroas. Após aprovação foi realizada a impressão dos modelos, fresagem das coroas em cera, prova das coroas em cera e fresagem em cerâmica das coroas. Com base na experiência vivenciada parece lícito concluir que os desenvolvimentos tecnológicos têm tornado as reabilitações odontológicas complexas mais rápidas, mais efetivas e previsíveis.
\end{abstract}


Palavras-chave: Tomografia computadorizada de feixe cônico; CAD-CAM; Reabilitação bucal; Implantes dentários; Impressão tridimensional.

\begin{abstract}
Rehabilitation dentistry is undergoing a major evolution in all areas of knowledge, whether through scientific research, development of surgical techniques and development of materials. With the emergence of new technologies, more resources are being added to the daily clinic, since materials and techniques help from diagnosis to the manufacture of prostheses on implants. The objective of this clinical case report is to demonstrate the importance of associating several areas of knowledge in implant-supported rehabilitation, valuing the benefits of using cone beam computed tomography, the CAD / CAM system, intraoral scanners and adhesive techniques in rehabilitation. A 54year-old patient sought treatment at Fundecto-USP (Fundação Faculdade de Odontologia associated with FOUSP) with a complaint of dental absence in the region of elements 14 and 15, in addition to complaints regarding aesthetics in the anterior region. The diagnosis was made through assembly in a semi-adjustable articulator, photographs and diagnostic waxing. Despite the absence of two elements, rehabilitation with implantation of only element 15 and an increase in elements 16 and 13 was planned. Cone beam computed tomography allowed to identify the absence of sufficient bone height for implant installation in the region of 15, so it was planned installation of the implant with simultaneous lifting of the maxillary sinus membrane. Through intraoral scanning, CAD / CAM technology and clinical information, the virtual waxing project for future crowns was carried out. After approval, the models were printed, reamed the wax crowns, tasted the wax crowns and ceramic reamed crowns. Based on the experience, it seems reasonable to conclude that technological developments have made complex dental rehabilitation faster, more effective and predictable.
\end{abstract}

Keywords: Cone-beam computed tomography; Computer-aided design; Mouth rehabilitation; Dental implants; Three-dimensional printing.

\title{
Resumen
}

La odontología rehabilitadora está experimentando una importante evolución en todas las áreas del conocimiento, ya sea a través de la investigación científica, el desarrollo de técnicas quirúrgicas y el desarrollo de materiales. Con la irrupción de las nuevas tecnologías se van sumando más recursos a la clínica diaria, ya que los materiales y técnicas ayudan desde el diagnóstico hasta la fabricación de prótesis sobre implantes. El objetivo de este caso clínico es demostrar la importancia de asociar varias áreas de conocimiento en la rehabilitación implantosoportada, valorando los beneficios del uso de la tomografía computarizada de haz cónico, el sistema CAD / CAM, escáneres intraorales y técnicas adhesivas en rehabilitación. Un paciente de 54 años buscó tratamiento en la Fundecto-USP (Fundação Faculdade de Odontologia asociada a FOUSP) con una queja de ausencia dentaria en la región de los elementos 14 y 15, además de quejas relacionadas con la estética en la región anterior. El diagnóstico se realizó mediante montaje en articulador semi-ajustable, fotografías y encerado diagnóstico. A pesar de la ausencia de dos elementos, se planificó la rehabilitación con implantación de solo el elemento 15 y aumento de los elementos 16 y 13. La tomografía computarizada de haz cónico permitió identificar la ausencia de altura ósea suficiente para la instalación del implante en la región de 15, por lo que se planificó instalación del implante con elevación simultánea de la membrana del seno maxilar. Mediante escaneo intraoral, tecnología CAD / CAM e información clínica se llevó a cabo el proyecto de encerado virtual para futuras coronas. Después de la aprobación, los modelos se imprimieron, se escariaron las coronas de cera, se probaron las coronas de cera y las coronas de cerámica escariadas. A partir de la experiencia, parece razonable concluir que los avances tecnológicos han hecho que la rehabilitación dental compleja sea más rápida, eficaz y predecible.

Palabras clave: Tomografía computarizada de haz cónico; Diseño asistido por computadora; Rehabilitación bucal; Implantes dentales; Impresión tridimensional.

\section{Introdução}

A odontologia reabilitadora está passando por uma grande evolução em todas as áreas de conhecimento, através das pesquisas cientificas e evolução tecnológica. Em um planejamento de reabilitação oral, o cirurgião-dentista precisa estar atento não só à reposição dos elementos dentários ausentes, mas na sua interrelação com os demais elementos e contribuir de forma a restabelecer a harmonia estética, funcional e biológica de todo o sistema estomatognático (Magda-Ecaterina, Monica, Ramona, Ovidiu \& Consuela, 2018; Condurache, Matei, Boancă, Buga \& Earar, 2020; Marcelino et al., 2020).

O planejamento prévio da reabilitação oral, também chamado de planejamento reverso, se faz extremamente necessário antes de qualquer intervenção reabilitadora. E, quando associado às novas tecnologias disponíveis, permitem resultados clínicos mais seguros e estáveis (Condurache et al., 2020; Gondim et al., 2021). Portanto, o cirurgião-dentista deve 
ter conhecimento das expectativas e necessidades do tratamento, como também as particularidades de cada caso e, a partir disso, planejar as etapas necessárias para a sua execução.

Cientes dessa necessidade, compreende-se que buscar esses novos recursos tecnológicos pode ser importante para elaborar um bom planejamento em reabilitação oral. Após o diagnóstico, onde exames de imagem digital, sejam radiográficos ou tomográficos, podem nos mostrar imagens tridimensionais de toda estrutura óssea, o cirurgião-dentista pode realizar todo o planejamento cirúrgico e protético através de um worflow totalmente digital. Imagens obtidas através de escaneamento intraoral podem ser compartilhadas com o laboratório e o planejamento e confecção das próteses podem ser realizadas pelo processo CAD/CAM (Beldiman, Tatarciuc, Luca \& Vițalariu, 2020; Gondim et al., 2021).

Desta forma, este trabalho tem como objetivo descrever o uso de alguns recursos tecnológicos disponíveis, como: tomografia digital, escaneamento intra-oral e processos CAD/CAM, no planejamento e execução das reabilitações dento e implantossuportadas, evidenciando suas aplicações clínicas e benefícios através de um estudo de caso.

\section{Metodologia}

Este trabalho trata-se de um estudo de caso, descritivo e qualitativo. De acordo com Pereira, Shitsuka, Parreira \& Shitsuka (2018) este tipo de estudo refere-se a uma descrição de uma temática específica, esmiuçando-a para evidenciar suas particularidades e qualificar sua relevância. No trabalho em questão, evidencia-se os avanços tecnológicos aplicados à reabilitação oral implantossuportada. Em relação aos aspectos éticos, foram fornecidas informações ao paciente por meio do Termo de Consentimento Livre e Esclarecido (TCLE) e a autorização do procedimento ocorreu mediante assinatura deste documento.

\section{Estudo de Caso}

Paciente, 54 anos, gênero feminino, procurou tratamento na Fundecto-USP (Fundação Faculdade de Odontologia conveniada à FOUSP) com queixa de ausência dentária na região dos elementos 14 e 15, além de queixa estética na região anterior.

Ao exame clínico inicial, observou-se ausência dentária dos elementos 14 e 15 com mesialização dos elementos 16,17 e 18 com espaço mésio distal reduzido para os elementos 14 e $15(12 \mathrm{~mm})$ e presença de oclusão em topo na região anterior, como pode ser observado na Figura 1. Observa-se também, depressão na região vestibular dos pré-molares acima referidos, sugerindo reabsorção óssea horizontal por atrofia da cortical vertical vestibular.

Figura 1 - Aspecto clínico inicial da paciente. A: Imagem da oclusão da paciente, onde observa-se uma mordida em topo, ausência dos elementos 14 e 15 e atrofia alveolar na região; B: Observa-se com mais detalhes a região anterior, onde visualizase dentes com facetas de desgaste, alteração de cor e forma.

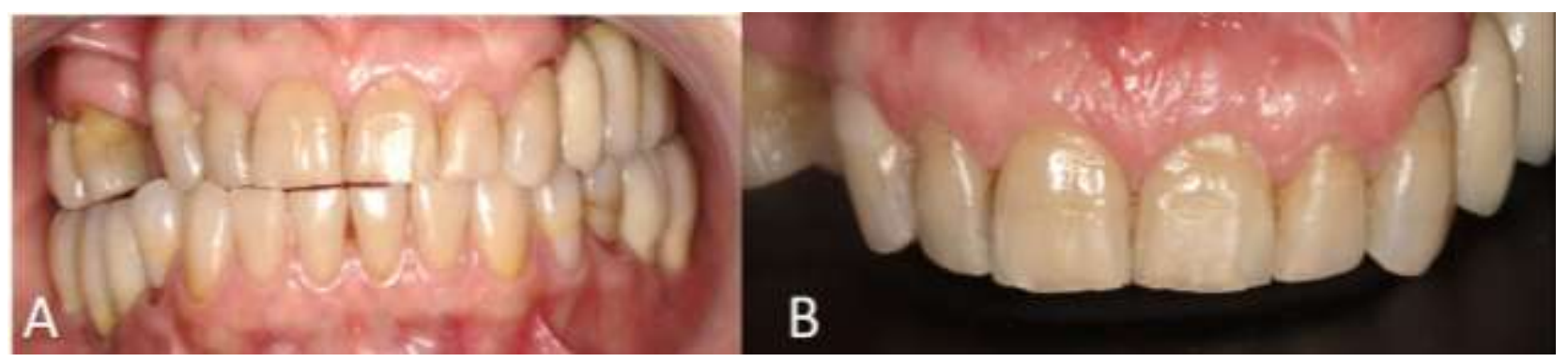

Fonte: Autores (2021). 
Para confirmar as impressões clínicas, foram obtidos os modelos de trabalho e realizada a montagem deles em articulador semi-ajustável. Foi solicitada a documentação fotográfica e tomografia computadorizada. A análise facial foi realizada para observação da dimensão vertical, suporte de lábio, proporção facial e a exposição de incisivo superior em repouso e sorriso espontâneo. Os modelos montados foram enviados ao laboratório de prótese dentária para realização de enceramento diagnóstico, com sugestão de suprimir o dente 15, aumentar a mesial do elemento 16, aumentar a distal do elemento 13 e dessa forma alcançar um pré-molar de dimensões normais e compatíveis com o padrão dentário da paciente. Também foi solicitado enceramento diagnóstico, na Figura 2, dos dentes 13 a 23 com recuperação de forma e comprimento, para obter mais exposição de dentes anteriores e consequentemente melhoria estética.

Figura 2 - Enceramento diagnóstico, no qual foi melhorado a forma e tamanho dos elementos dentários, com aumento da mesial do elemento 16 e a distal do elemento 13, com intuito de deixar um espaço protético compatível a coroa de um prémolar (elemento 14).

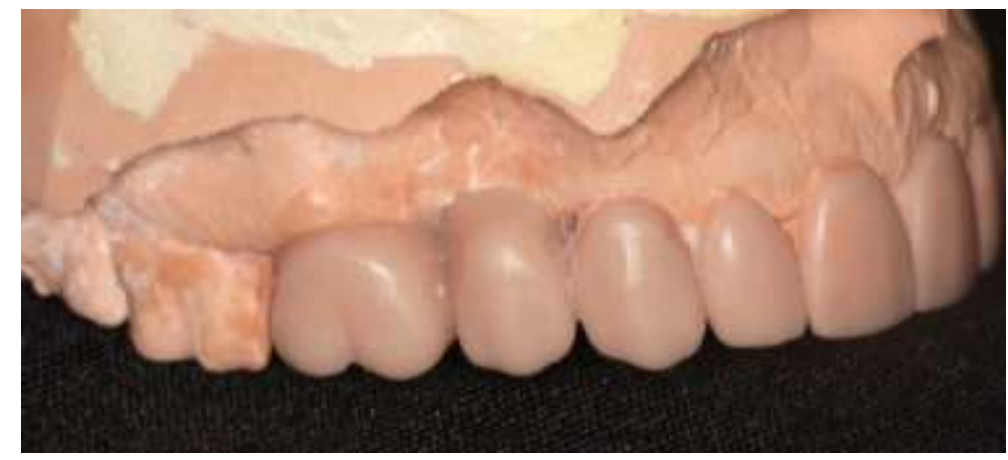

Fonte: Autores (2021).

Para realizar o ensaio restaurador (Mock-up), confeccionou-se uma guia sobre o modelo de estudo encerado em silicone de adição, pesada e leve, como observado na Figura 3. A resina bisacrílica (structor 2 sc A2- VOCO) foi inserida no guia para Mock-up e foi levada em boca em sua posição exata. Após a remoção dos excessos e ajustes dos contatos e guias de desoclusão, optou-se por deixar o ensaio restaurador, na Figura 4, por 48 horas para avaliação estético-funcional mais precisa. Após esse período teve-se a aprovação do tratamento.

Figura 3 - Modelo encerado e Guia de Mock-up, no qual será colocado a resina bisacrílica e levado a boca da paciente para simular a reabilitação que está sendo planejada e assim realizar os ajustes necessários no planejamento.

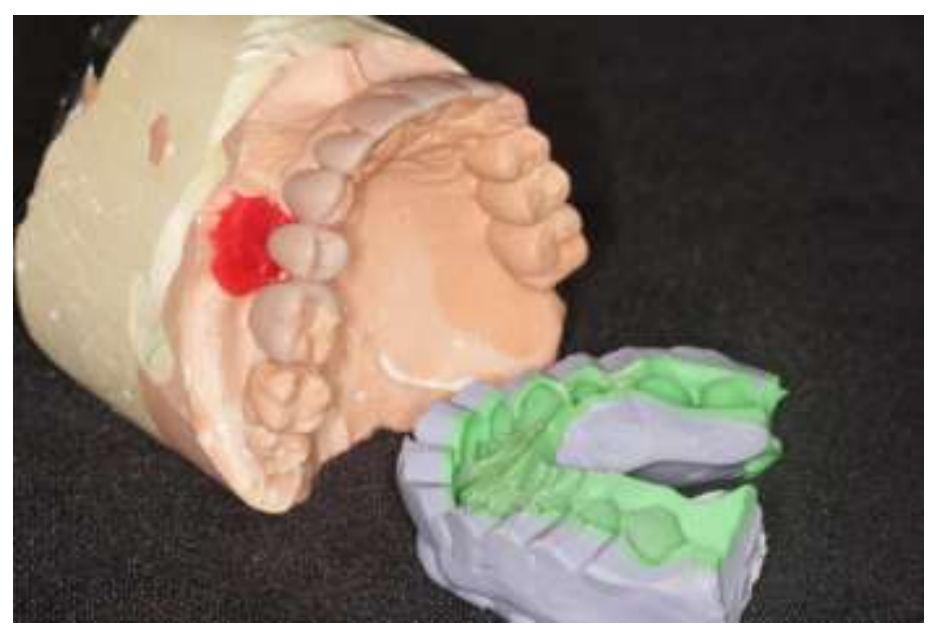

Fonte: Autores (2021). 
Figura 4 - Mock-up realizado.

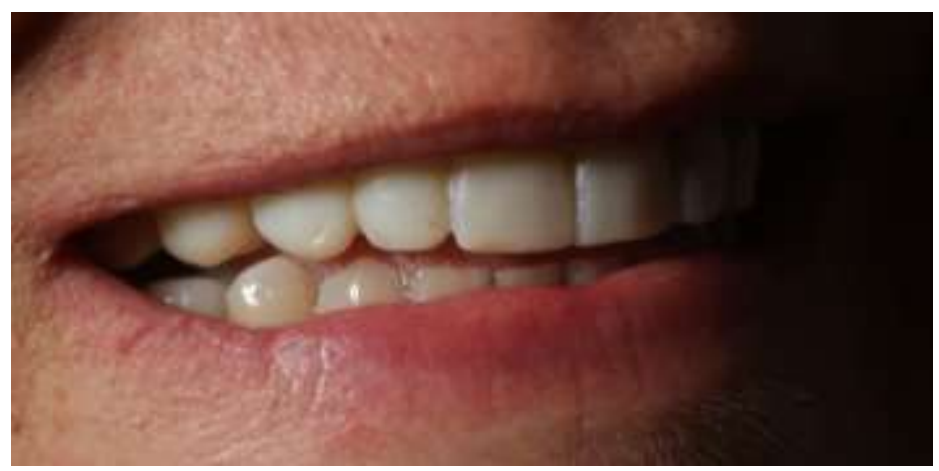

Fonte: Autores (2021).

Foi solicitado o exame tomográfico, que está disponível na Figura 5, para avaliação do remanescente ósseo na região e planejamento da reabilitação implantossuportada. O exame revelou altura e espessura reduzidas para realização do implante, reforçando o diagnóstico clínico e sugerindo o enxerto ósseo para ganho em espessura e altura.

Figura 5 - Cortes tomográficos, evidenciando altura e espessura ósseas reduzidas na região dos cortes 14 e 15.

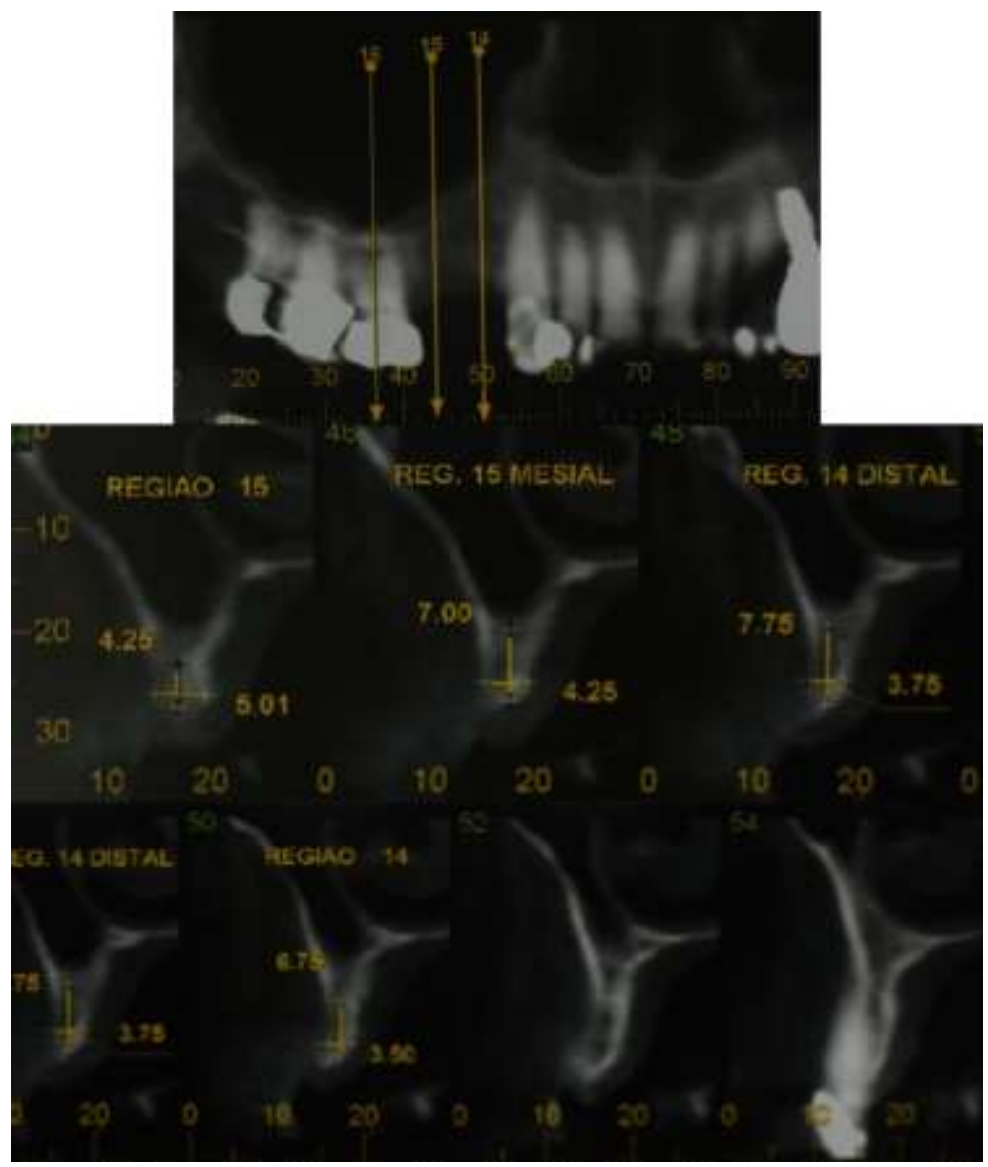

Fonte: Autores (2021).

Na Figura 6, observa-se o acesso à parede lateral do seio maxilar nas posições dos dentes 14 e 15 para levantamento da membrana do seio maxilar. O preenchimento foi realizado com Bio-Oss ${ }^{\circledR}$ Large (Geistlich, Wolhusen, Suiça). Em seguida, utilizando um guia cirúrgico adaptado sobre os dentes, foi realizada a fresagem e instalação de um implante Titamax cortical CM (Neodent, Curitiba, Brasil) com dimensões de 3.75X13mm (torque de 45 Ncm), simultaneamente à elevação do assoalho 
do seio maxilar. Foi também realizado enxerto para ganho em espessura, utilizando o mesmo biomaterial da elevação do seio maxilar e a proteção foi realizada com membrana Bio-Gide ${ }^{\circledR}$ (Geistlich, Wolhusen, Suiça).

Figura 6 - Levantamento do seio maxilar simultaneamente à instalação de implante dentário. A: Vista frontal evidenciando o paralelismo da perfuração inicial com o guia em posição; B: Região de levantamento de seio maxilar com implante em posição; C: Enxerto realizado para ganho de espessura óssea vestibular; D: Enxerto recoberto com membrana de colágeno.
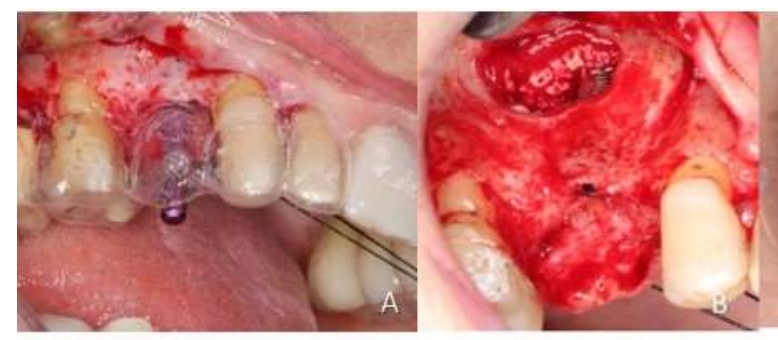
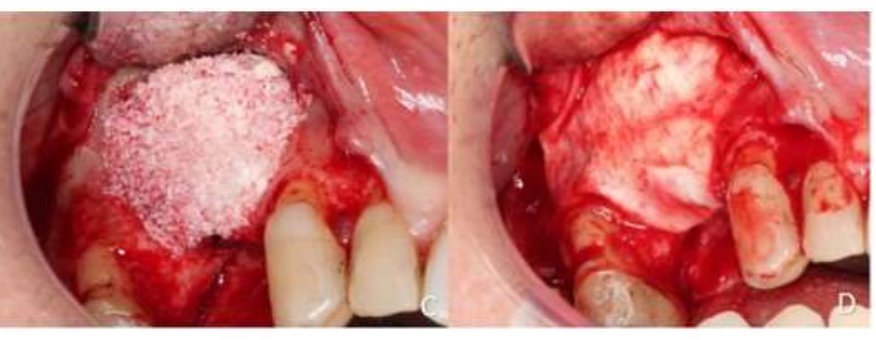

Fonte: Autores (2021).

Após 7 meses, a paciente apresentou queixa álgica na região do elemento 16 e foi verificado lesão cariosa na distal do dente 16 com comprometimento radicular. Foi realizada a exodontia do elemento 16 com instalação imediata de implante Drive CM Aqua (Neodent, Curitiba, Brasil) com dimensões de 4.3X11,5 (torque de 60Ncm), como observado na Figura 7. Em virtude da integração do implante da região do 14 e torque de $60 \mathrm{Ncm}$ do implante da região do 16 , foi indicado a provisionalização imediata. Os componentes protéticos foram selecionados com Kit protético cone-Morse (Neodent). Os intermediários escolhidos foram os minipilares cônicos (Neodent). Foi aplicado o torque recomendado pelo fabricante (32 $\mathrm{Ncm}$ ) para ambos pilares retos selecionados. Foram adaptados 2 cilindros de titânio sobre os minipilares para confecção das coroas provisórias em resina acrílica e dentes de estoque, unidos e parafusados.

Figura 7 - Exodontia do 16 com implante imediato e provisonalização. A: Mostra o paralelismo entre os implantes do 16 e da região do 14 realizado previamente; B: Instalação do implante na região do 16 com torque de $60 \mathrm{Ncm}$; C: Vista lateral das coroas provisórias instaladas.

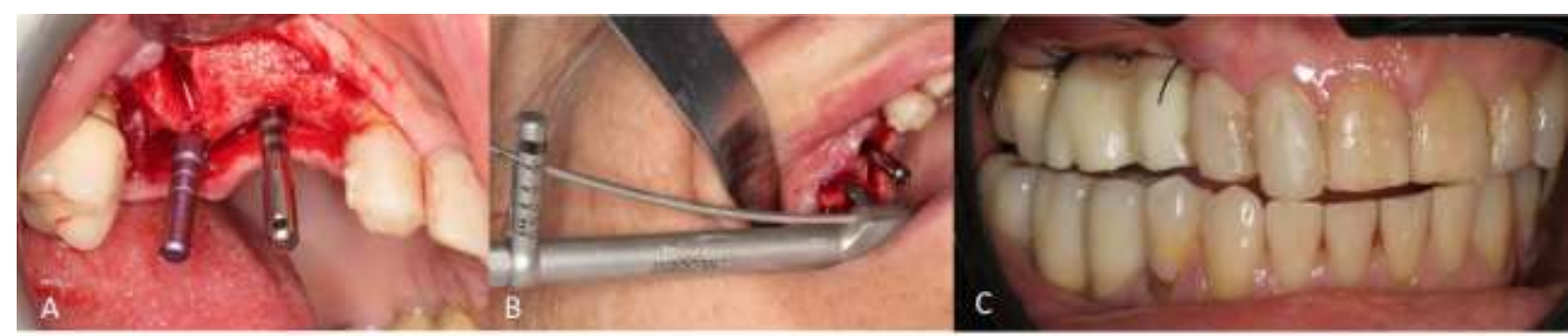

Fonte: Autores (2021).

Após 4 meses de pós operatório e condicionamento gengival foi dado início à finalização do caso, foi optado por seguir com fluxo de trabalho digital (workflow digital). Na Figura 8, observa-se que os elementos dentários 11 e 21 receberam preparos para faceta, os elementos 13, 12, 22 e 23 receberam preparo para coroa total, todas em dissilicato de lítio. Os preparos foram realizados com brocas diamantadas e multilaminadas em alta rotação (Kavo) e contra ângulo multiplicador 1:5 (Sirona) respectivamente, acabamento final com discos soflex (3M). 
Figura 8 - Preparos realizados nos elementos 13 a 23. A: Evidenciando os preparos para coroa nos elementos $13,12,22$ e 23 e para faceta nos elementos 11 e 21; B: Mostrando de forma mais detalhada os preparos dos elementos 12 ao 22.

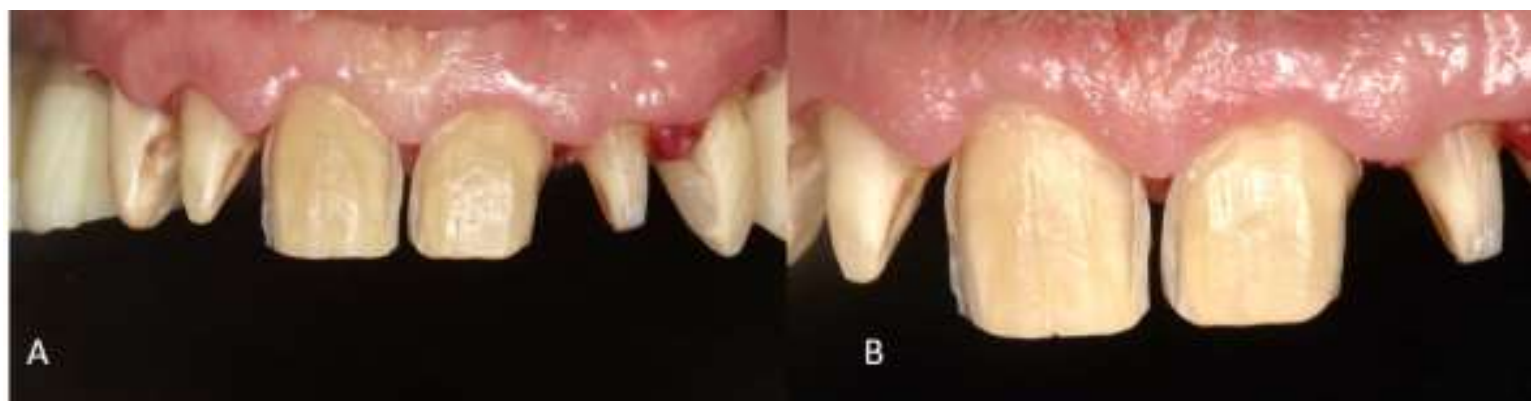

Fonte: Autores (2021).

Na Figura 9, utilizando a técnica de afastamento gengival com fio retrator (Pegoraro 1998), realizamos a moldagem digital utilizando um scanner intraoral (Carestream). Foram escaneados os preparos dos elementos 13 a 23 após a remoção dos fios retratores gengivais e em sequência foram escaneados os transfer's digitais (região dos dentes 14 e 16) adaptados sobre os minipilares (Neodent), formando uma única imagem da maxila. Posteriormente foi realizado o escaneamento do arco antagonista e escaneamento das arcadas em oclusão para montagem dos modelos virtuais e registro oclusal virtual.

Figura 9 - Modelos digitais obtidos a partir de escaneamento intraoral (Carestream). A: Modelo digital do arco superior, região anterior e posterior da maxila, onde observa-se os transfer's digitais na região do 15 e 16; B: Modelos digitais em oclusão.

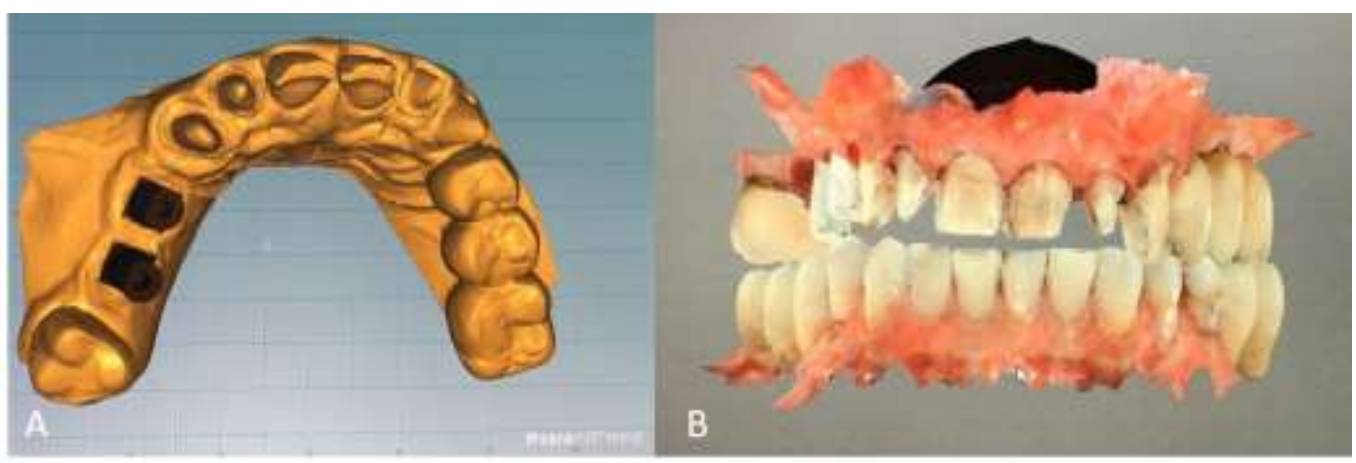

Fonte: Autores (2021).

De posse dos modelos virtuais, da documentação fotográfica da paciente e das informações clínicas transmitidas pelos profissionais responsáveis pela reabilitação, o desenhista de CAD-CAM (Ceramill Mind, AmannGirrbach, Curitiba, Brasil) desenvolveu o projeto de enceramento virtual nos referidos dentes como observado na Figura 10. 
Figura 10 - Enceramento virtual. A: Vista frontal do enceramento da região anterior de maxila; B: Vista lateral onde evidencia-se o enceramento das coroas dos elementos dentários 15 e 16; C:Vista frontal onde pode-se observar a oclusão da paciente e o enceramento de todos os elementos a serem reabilitados; D: Vista oclusal da maxila, mostrando o enceramento das coroas dos elementos 16, 15, 13, 12, 22 e 23, como também das facetas do 11 e 21.

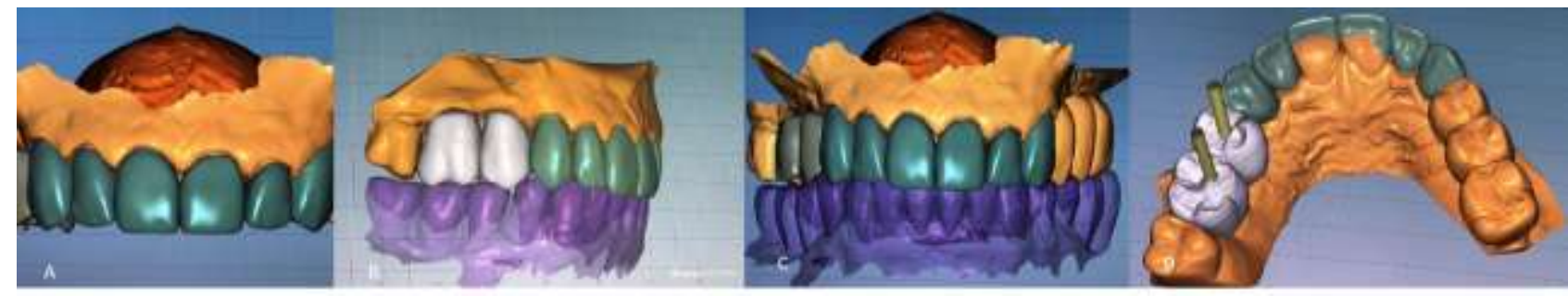

Fonte: Autores (2021).

Após a aprovação do enceramento virtual, foi realizada a impressão do modelo 3D e fresagem das facetas e coroas (Ceramill Motion, AmannGirrbach, Curitiba, Brasil) dos elementos 13 a 23 em cera para prova na paciente. As coroas provisórias foram removidas e então foi realizada a prova das facetas e coroas em cera. Foram confirmados os pontos de contato, o eixo de inserção, a adaptação das peças aos preparos e as margens de términos, como na Figura 11. Novas facetas provisórias foram confeccionadas com o Mock-up e resina bisacrílica. Após a prova, as facetas foram incluídas e levadas para injeção com dissilicato de lítio (e-max Press - Ivoclar, Schaan, Liechtenstein) e posterior adaptação ao modelo impresso. As maquiagens foram realizadas e o glaze final, evidenciada na Figura 12.

Figura 11 - Modelo impresso e prova das coroas e facetas da região anterior. A: Modelo impresso; B: Vista frontal das coroas e facetas da região anterior; C: Vista palatina das coroas e facetas da região anterior.

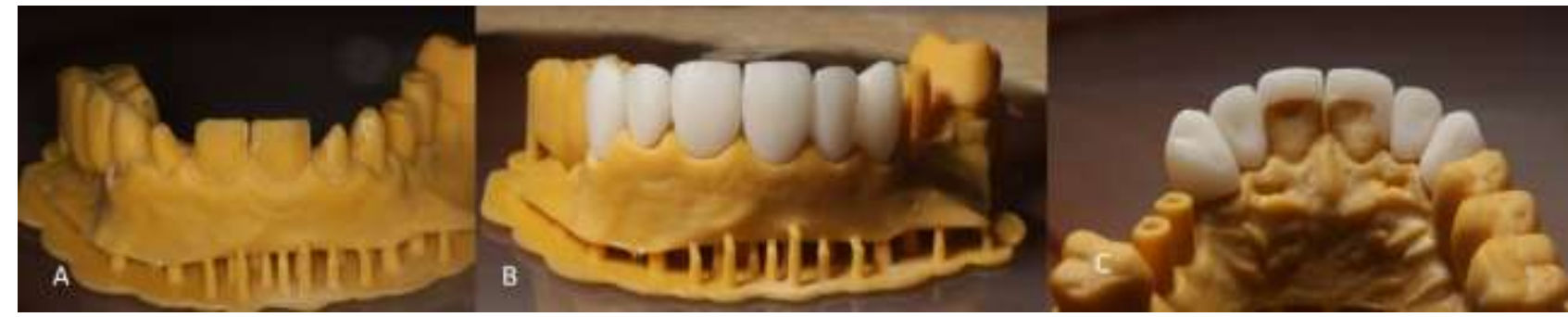

Fonte: Autores (2021).

Figura 12 - Coroas e facetas finalizadas em cerâmica.

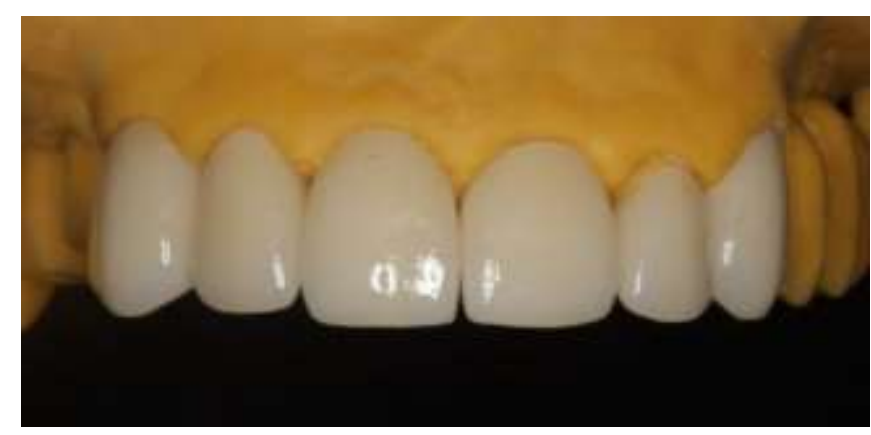

Fonte: Autores (2021).

Na sessão para cimentação das peças, na Figura 13, as peças provisórias foram removidas e os preparos foram higienizados com escova e pedra pomes. As peças receberam o tratamento preconizado: ácido fosfórico à $37 \%$, esfregaço 
delicado com micro-brush por 1 minuto e em seguida lavados com água por igual período; : ácido fluorídrico à 10\% por 20 segundos e em seguida lavados com água por igual período; aplicação de silano (Monobond N - Ivoclar) em dupla camada por 1 minuto. Os dentes preparados foram condicionados com ácido fosfórico à 37\% por 15 segundos em dentina e 30 segundos em esmalte sendo lavados por 30 segundos. Em seguida, o adesivo (Tetric $\mathrm{N}$ bond Universal - Ivoclar Vivadent) foi aplicado nas superfícies dos preparos e nas peças por 20 segundos sendo esfregados. Após aplicação de ar gentilmente por 20 segundos nos dentes e nas facetas, o cimento Variolink Esthetic (Ivoclar Vivadent) cor neutral foi colocado nas facetas. Elas foram adaptadas sobre os preparos, os excessos de cimento foram removidos foi realizada a polimerização (Bluephase Ivoclar Vivadent) por 40 segundos em cada face, vestibular e palatina respectivamente. Na Figura 14 observam-se as peças da região anterior cimentadas em boca.

Figura 13 - Cimentação das peças. A: Limpeza das peças com ácido fosfórico 37\%; B: Condicionamento ácido das peças com ácido fluorídrico a 10\%; C: Aspecto das peças após aplicação do ácido fluorídrico a 10\%; D: Aplicação do Silano; E: Condicionamento ácido dos dentes com ácido fosfórico 37\%; F: Aplicação do adesivo; G: Peça sendo levada à boca; H: Fotopolimerização; I: Finalização da cimentação.

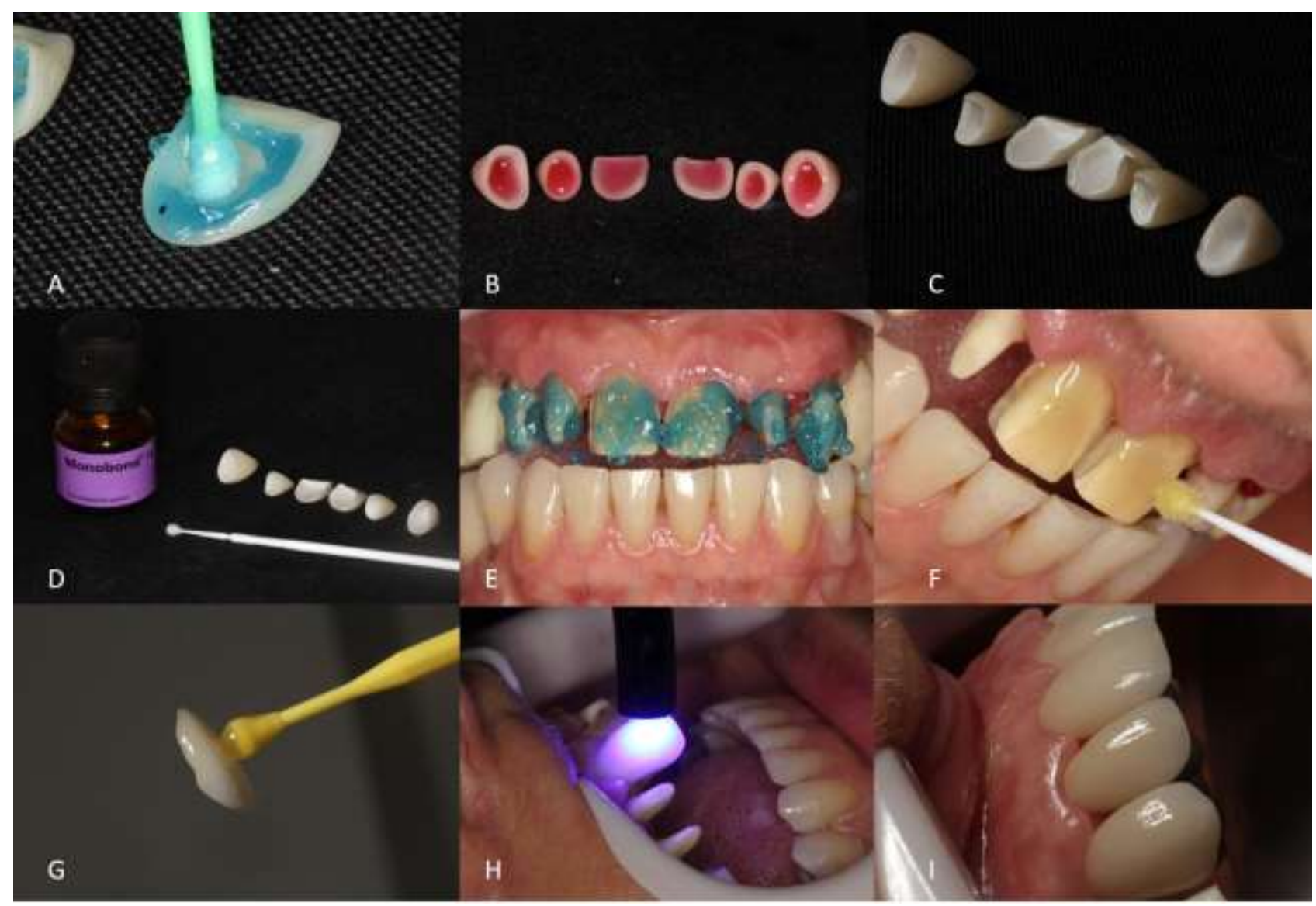

Fonte: Autores (2021). 
Figura 14 - Vista frontal da cimentação das peças da região anterior finalizada.

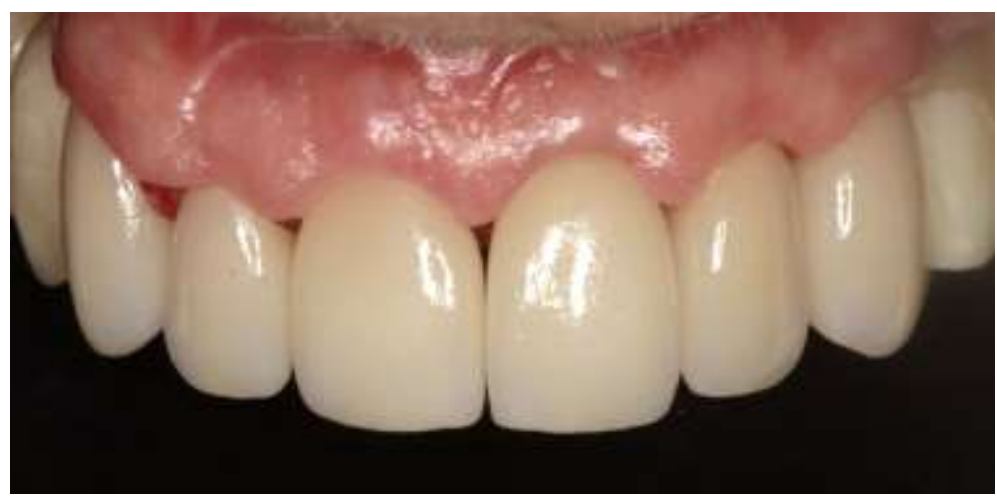

Fonte: Autores (2021).

A infraestrutura metálica da prótese sobre implante dos elementos 14 e 16 foi provada e foi realizado um registro a mordida com resina acrílica, como mostrado na Figura 15.

Figura 15 - A: Infraestrutura metálica da prótese sobre implantes; B: Prova da infraestrutura metálica; C: Aspecto radiográfico da infraestrutura em posição; D: Registro de oclusão em resina acrílica.
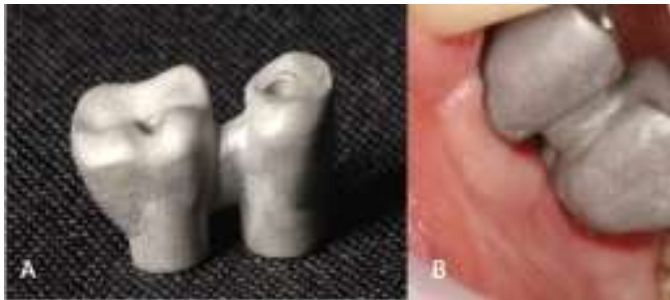
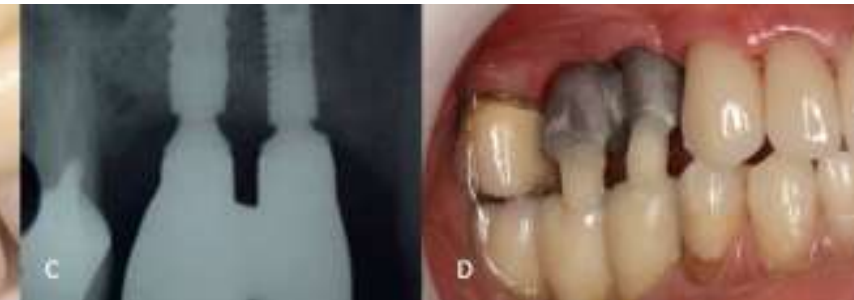

Fonte: Autores (2021).

O trabalho retornou ao laboratório para aplicação da cerâmica e glaze final. A prótese implantossuportada foi instalada com torque de $10 \mathrm{Ncm}$ nos parafusos de minipilar, Foi realizada proteção dos parafusos protéticos com teflon e os orifícios foram restaurados com resina composta. Realizou-se ajuste oclusal de refinamento para contatos oclusais, guias laterais de desoclusão e anterior. A Figura 16 mostra o caso finalizado. A paciente teve sua estética e função restauradas, tendo melhora da sua autoestima e qualidade de vida.

Figura 16 - Finalização do caso. A: Vista frontal da arcada reabilitada; B: Vista palatina evidenciando o término da faceta nos dentes 11 e 21 e o remanescente dentário; C: Vista lateral do sorriso, mostrando a melhora no overbite, estética e naturalidade; D: Vista frontal do sorriso.

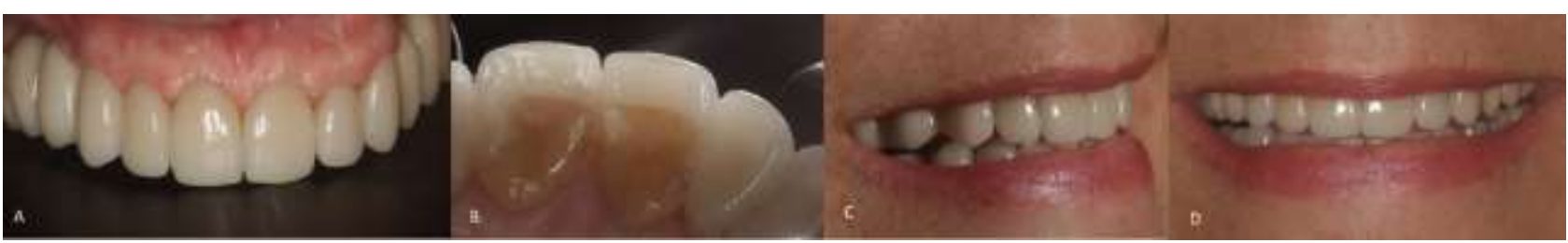

Fonte: Autores (2021). 


\section{Discussão}

A exigência estética dos pacientes e a busca pela excelência das reabilitações orais vem aumentando nos últimos anos (Medeiros et al., 2020; Marcelino et al., 2020). Isto é válido, tanto para os casos de pacientes que procuram por restaurações estéticas, como também para os casos que buscam tratamentos para reposição de poucos elementos ou até mesmo de arcos totais edêntulos. Assim sendo, a necessidade de atenção por parte do profissional em relação ao desejo dos pacientes torna-se cada vez mais importante na indicação do tipo de tratamento que trará mais satisfação. Essa necessidade de oferecer melhores possibilidades de tratamentos clínicos exige que o profissional busque, além de capacitação pessoal, o trabalho em conjunto com outros profissionais de distintas especialidades (Gondim et al., 2021).

O conhecimento das novas técnicas que vem sendo disponibilizadas graças aos avanços tecnológicos também se torna importante para elevar a qualidade dos tratamentos. Essa interação entre especialidades e diversas áreas de conhecimento da odontologia tem sido recomendada (Bernardes, de Matias \& Thomé 2012; Gondim et al. 2021) e está demonstrada na resolução clínica aqui apresentada. Procedimentos cirúrgicos associados a protéticos permitiram a reabilitação e a melhora da auto estima da paciente.

A reabilitação oral com implantes exige uma íntima relação entre as especialidades, onde todas vão ter a participação no diagnóstico, planejamento e execução do caso (da Silva et al., 2020; de Macêdo et al., 2020; Gondim et al., 2021). A necessidade de atenção para elaborar um tratamento reabilitador sobre implantes tem sido considerada. Todo o planejamento deste caso apresentado foi elaborado sob o protocolo de planejamento reverso, onde se adota a previsão da reabilitação protética, a fim de orientar o planejamento cirúrgico a garantir que a execução seja a mais favorável possível para a futura prótese (Amoroso et al., 2012) O estudo do espaço edentulo permitiu observar a falta de espaço para reposição de dois elementos. $\mathrm{O}$ enceramento envolvendo aumento das dimensões proximais dos dois elementos vizinhos permitiu validar uma possibilidade de resolução. As fotos clínicas permitiram a complementação da coleta dos dados de necessidade de reposição estética. Notou-se que a falta de volume vestibular precisava ser solucionada.

Para seguir com o planejamento cirúrgico, os modelos de estudo foram utilizados para confecção de guias cirúrgicosradiográficos. A análise dos exames de imagem como radiografia panorâmica e tomografia computadorizada Cone Beam, para então ter condições de melhor executar as instalações dos implantes, e a cirurgia simultânea de levantamento do assoalho de seio maxilar (sinus lift) se revestiu de importância, uma vez que permitiu um ganho de tempo de tratamento. Foi também planejada a instalação prévia do implante na região 15 e só após a osseointegração do mesmo, o elemento 16 foi extraído e substituído pelo implante, para que a técnica de carga imediata pudesse então ser realizada.

Apesar do início da condução do caso ter sido realizada utilizando modelos e enceramento físicos, o desenvolvimento tecnológico fez com que na fase protética o escaneamento intraoral se tornasse disponível. Assim, cientes da importância da participação multidisciplinar, viu-se vantagens na utilização da digitalização do caso clínico, condicionando a dinâmica do trabalho entre os profissionais das distintas áreas, como considerado em estudos (Van Noort, 2012).

Como o workflow digital permite uma interação entre os profissionais envolvidos e a agilidade e qualidade dos trabalhos protéticos e o fluxo de trabalho convencional tem sido considerado como possuidor de maiores fontes potenciais de erro (Miyazaki, Hotta, Kunii, Kuriyama \& Tamaki, 2009; Neves, Barbosa \& Bernardes, 2016). Decidiu-se adotar essa sistemática na reabilitação protética. Dentro da proposta de oferecer um tratamento reabilitador integrado e de excelência, optou-se por substituir as moldagens tradicionais pelo escaneamento intraoral, que oferece precisão e alta fidelidade às imagens, na possibilidade de trabalho integrado ao sistema CAD/CAM para a confecção das unidades protéticas (Van Noort, 2012; Camardella, Rothier, Camardella \& Chaves, 2014).

Considerando que os laminados cerâmicos são indicados quando o dente possui restaurações não tão extensas com a finalidade de uniformizar a cor ou a forma, fechar diastemas, corrigir giro versões e anatomia dos dentes quando estes 
apresentam desgastes, fraturas, cáries ou alteração de cor (Miyashita \& Oliveira, 2014). Neste caso clínico, optou-se pela utilização dessa técnica na região anterior de maxila. Havia a necessidade de ganho de comprimento nos quatro incisivos superiores para obedecer aos princípios oclusais e estéticos, além de que os dois incisivos centrais apresentavam boa quantidade de estrutura remanescente em esmalte, o que indicava o procedimento. Já nos demais elementos, como haviam restaurações mais extensas, adotou-se o preparo total mas com menor quantidade de desgaste se comparado aos preparos convencionais para coroas totais em metalocerâmica, uma vez que seria utilizado o dissilicato de lítio como material de eleição, e esse permite preparos mais econômicos (Van Noort, 2012).

Ao se utilizar o enceramento virtual para obtenção das próteses, foi avaliada a oclusão do caso no software. Como havia sido obtida a interrelação entre arcos no escaneamento, foram gerados os registros oclusais das arcadas, ou seja, um articulador digital. Assim, pode-se ver as áreas de contatos oclusais e a escala de intensidade, possibilitando planejar acréscimos e remoções de material, assim como ajuste oclusal em superfície dentária, se necessário.

\section{Considerações Finais}

Fundamentado na literatura consultada, na experiência clínica vivenciada e no nível de satisfação da paciente com o tratamento pode-se concluir que os desenvolvimentos tecnológicos têm tornado os tratamentos mais rápidos, mais efetivos e mais seguros, devolvendo estética, função, autoestima e qualidade de vida aos pacientes.

Com base na experiência vivenciada na execução do caso clínico e na execução desse relato, pode-se concluir que os recursos tecnológicos como as tomografias computadorizadas de feixe cônico, o escaneamento intraoral e o workflow digital com a utilização do sistema CAD/CAM são ferramentas, em evolução, que vieram contribuir e muito para o aprimoramento tanto do planejamento quanto da execução das reabilitações em implantodontia.

\section{Referências}

Amoroso, A. P., Gennari Filho, H., Pellizzer, E. P., Goiato, M. C., Júnior, S., Ferreira, J., \& Villa, L. M. R. (2012). Planejamento reverso em implantodontia: relato de caso clínico. Revista Odontológica de Araçatuba, 75-79.

Beldiman, M. A., Tatarciuc, M., Luca, E., \& Vițalariu, A. M. (2020). Technological aspects in cad/cam fixed rehabilitation on implants. Romanian Journal of Oral Rehabilitation, 12(2).

Bernardes, S. R., de Matias, T. S. I., \& Thomé, G. (2012). Tecnologia CAD/CAM aplicada a prótese dentária e sobre implantes. Jornal ILAPEO, 6(1), 8-13.

Camardella, L., Rothier, E. K. C., Camardella, E. G., \& Chaves, R. (2014). A utilização dos modelos digitais em Ortodontia. Ortodontia SPO, 47(1), 75-82.

Condurache, G. G., Matei, M., Boancă, A. C., Buga, V., \& Earar, K. (2020). Aesthetic aspects in oral rehabilitation correlated with access to nowadays equipment. Romanian Journal of Oral Rehabilitation, 12(2), 155-164.

da Silva, R. A. R., de Almeida Neto, L. F., Marcelino, K. P., Cardoso, L. C. L., Dantas, E. M., Barbosa, G. A. S., \& Gondim, A. L. M. F. (2020). Osteotomia segmentar da maxila em área estética para viabilizar reabilitação com implantes dentários: relato de caso. Research, Society and Development, 9(9), e594997525-e594997525.

de Macêdo, F. C., de Almeida Neto, L. F., Marcelino, K. P., Dantas, E. M., Dantas, W. R. M., Barbosa, G. A. S., \& Gondim, A. L. M. F. (2020). Tratamento cirúrgico de fratura de mandíbula após transposição do nervo alveolar inferior: relato de caso. Research, Society and Development, 9(9), e838998021e838998021.

Gondim, A. L. M. F., Miyashita, E. ., Sartori, I. A. de M. ., Traina, A. A., Neves, F. D. das ., Belo, H. D. S. ., Almeida Neto, L. F. de, Marcelino, K. P., Ferreira, I. J., \& Riesco, M. G. . (2021). Interdisciplinary treatment for complex aesthetic rehabilitation of the smile: a case report. Research, Society and Development, 10(1), e16310111565. https://doi.org/10.33448/rsd-v10i1.11565

Magda-Ecaterina, A., Monica, A., Ramona, F., Ovidiu, S., \& Consuela, F. N. (2018). Aspects of oral rehabilitation using removable dentures: esthetics and functionality. Romanian Journal of Oral Rehabilitation, 10(1), 133-139.

Marcelino, K. P., de Souza, J. A. N., Ribeiro, D. T., Dantas, E. M., Barbosa, G. A. S., Gondim, A. L. M. F., \& de Almeida Neto, L. F. (2020). Conduta cirúrgica após perfuração da membrana de Schneider durante sinus lift: relato de caso. Research, Society and Development, 9(10), e9959109425e9959109425.

Medeiros, M. S., Marcelino, K. P., de Oliveira Júnior, J. C., Pinheiro, N. C. G., Freire, J. C. P., Dantas, E. M., \& de Almeida Neto, L. F. (2020). Exodontia atraumática e implante imediato em área estética: relato de caso. Research, Society and Development, 9(9), e880997997-e880997997. 
Research, Society and Development, v. 10, n. 1, e57010112158, 2021

(CC BY 4.0) | ISSN 2525-3409 | DOI: http://dx.doi.org/10.33448/rsd-v10i1.12158

Miyashita, E., \& Oliveira, G. G. (2014). Odontologia estética: os desafios da clínica diária. Napoleão.

Miyazaki, T., Hotta, Y., Kunii, J., Kuriyama, S., \& Tamaki, Y. (2009). A review of dental CAD/CAM: current status and future perspectives from 20 years of experience. Dental materials journal, 28(1), 44-56.

Neves, F. D. D., Barbosa, G. A. S., \& Bernardes, S. R. (2016). Fundamentos da prótese sobre implantes. In Fundamentos da prótese sobre implantes (pp. 365365).

Pereira A.S. et al. (2018). Metodologia da pesquisa científica. https://repositorio.ufsm.br/bitstream/handle/1/15824/Lic_Computacao_Metodologia-Pesquisa-Cientifica.pdf?sequence=1

Van Noort, R. (2012). The future of dental devices is digital. Dental materials, 28(1), 3-12. 